\title{
A LOFAR census of non-recycled pulsars: extending to frequencies below $80 \mathrm{MHz}^{\star}$
}

\author{
A. V. Bilous ${ }^{1}$, L. Bondonneau ${ }^{2}$, V. I. Kondratiev ${ }^{3,4}$, J.-M. Grießmeier ${ }^{2,5}$, G. Theureau ${ }^{2,5,6}$, J. W. T. Hessels ${ }^{1,3}$, \\ M. Kramer ${ }^{7,8}$, J. van Leeuwen ${ }^{1,3}$, C. Sobey ${ }^{9}$, B. W. Stappers ${ }^{8}$, S. ter Veen ${ }^{3}$, and P. Weltevrede ${ }^{8}$ \\ 1 Anton Pannekoek Institute for Astronomy, University of Amsterdam, Science Park 904, 1098 Amsterdam, The Netherlands \\ e-mail: A.Bilous@uva.nl \\ 2 LPC2E - Université d'Orléans/CNRS, 45071 Orléans Cedex 2, France \\ 3 ASTRON, the Netherlands Institute for Radio Astronomy, Postbus 2, 7990 Dwingeloo, The Netherlands \\ ${ }^{4}$ Astro Space Centre, Lebedev Physical Institute, Russian Academy of Sciences, Profsoyuznaya Str. 84/32, 117997 Moscow, Russia \\ 5 Station de Radioastronomie de Nançay, Observatoire de Paris, PSL Research University, CNRS, Univ. Orléans, OSUC, 18330 \\ Nançay, France \\ ${ }^{6}$ Laboratoire Univers et Théories LUTh, Observatoire de Paris, CNRS/INSU, Université Paris Diderot, 5 Place Jules Janssen, 92190 \\ Meudon, France \\ 7 Max-Planck-Institut für Radioastronomie, Auf dem Hügel 69, 53121 Bonn, Germany \\ 8 Jodrell Bank Centre for Astrophysics, School of Physics and Astronomy, University of Manchester, Manchester M13 9PL, UK \\ 9 CSIRO Astronomy and Space Science, PO Box 1130, Bentley 6102, Australia
}

Received 3 September 2019 / Accepted 17 October 2019

\begin{abstract}
We present the results from the low-frequency $(40-78 \mathrm{MHz})$ extension of the first pulsar census of non-recycled pulsars carried out with the LOw-Frequency ARray (LOFAR). We used the low-band antennas of the LOFAR core stations to observe 87 pulsars out of 158 that had been previously detected using high-band antennas. We present flux densities and flux-calibrated profiles for the 43 pulsars we detected. Of this sample, 17 have not, to our knowledge, previously been detected at such low frequencies. Here we recalculate the spectral indices using the new low-frequency flux density measurements from the LOFAR census and discuss the prospects of studying pulsars at very low frequencies using current and upcoming facilities, such as the New Extension in Nançay Upgrading LOFAR (NenuFAR).
\end{abstract}

Key words. pulsars: general

\section{Introduction}

Half a century ago, work on interplanetary scintillation at the frequency of $81.5 \mathrm{MHz}$ led to the serendipitous discovery of pulsars (Hewish et al. 1968). Until recently, however, most pulsar observations were conducted at higher frequencies of 300$3000 \mathrm{MHz}$. Properties of pulsar emission at radio frequencies below $200 \mathrm{MHz}$ have remained relatively poorly explored for two reasons: the high level of background Galactic emission and the deleterious influence of electron plasma in the interstellar medium (ISM) and Earth's ionosphere.

The last decade has brought rapid advances both in hardware and computing capabilities, allowing unprecedented possibilities for sensitive broadband observations of pulsars with precise compensation for dispersive delay at frequencies below $200 \mathrm{MHz}$. These observations deepen our understanding of pulsars as astrophysical objects: for example, the frequencydependent spectral shape of radio emission and the morphology of the average pulse shape provide information about the microphysics of pulsar radio emission and magnetospheric configurations. In addition, because of their increased effects on the received signal at lower frequencies, the ISM and the ionosphere can be studied more accurately.

\footnotetext{
* The profiles are also available at the CDS via anonymous $\mathrm{ftp}$ to cdsarc.u-strasbg. fr (130.79.128.5) or via http://cdsarc. u-strasbg.fr/viz-bin/cat/J/A+A/635/A75
}

The new generation of low-frequency radio telescopes has already started charting the meter-wavelength pulsar sky. Several surveys of the known pulsar population have been conducted over the last few years. The newly-upgraded second modification of the Ukrainian T-shaped radio telescope (UTR-2) was utilised to detect 40 pulsars at $10-30 \mathrm{MHz}$, the lowest radio frequencies visible from Earth (Zakharenko et al. 2013). The first station of the Long Wavelength Array (LWA1) was used to measure the flux densities of 44 pulsars at 30-88 MHz (Stovall et al. 2015). At $185 \mathrm{MHz}$, the Murchison Widefield Array (MWA) was used to detect 50 pulsars (including six millisecond pulsars, Xue et al. 2017) and also to measure flux densities from continuum images (Murphy et al. 2017).

In 2014, we undertook a large campaign of observing almost all known non-recycled radio pulsars with declination (Dec) Dec $>8^{\circ}$ and Galactic latitude, $(\mathrm{Gb})|\mathrm{Gb}|>3^{\circ}$. The observations were performed with the high-band antennas (HBA) of the LOFAR telescope at frequencies of $110-188 \mathrm{MHz}$ (van Haarlem et al. 2013). The census (hereafter, the HBA census) encompassed 194 such sources and resulted in 158 detections, updating dispersion measures (DM) and measuring flux density values (Bilous et al. 2016, hereafter B16). Based on the measurements at $110-188 \mathrm{MHz}$ and the previously published flux densities, we constructed the broadband spectra and measured the spectral indices with a single or broken power-law model. It appeared that the spectra of most pulsars are, in fact, 
not very well known and regular flux density measurements are needed as flux densities can typically vary up to an order of magnitude due to diffractive and refractive interstellar scintillation or due to intrinsic variability. With the exception of a handful of bright pulsars with hundreds of flux density measurements, the choice of the model and the frequency of the spectral turnover depends in great measure on the insufficiently explored lowfrequency end of the spectrum.

To investigate the shape of the pulsar spectra further, we undertook an low-band antenna (LBA) extension of the HBA census (hereafter, the LBA census) encompassing 87 out of 158 pulsars that have been detected in the HBA census. This paper presents the average profiles, DMs, and flux density measurements for the pulsars that were detected. The results presented will be also made available through the European Pulsar Network (EPN) Database for Pulsar Profiles ${ }^{1}$.

\section{Source selection}

For the HBA census, we selected pulsars from version 1.51 of the ATNF Pulsar Catalogue ${ }^{2}$ which satisfied the following criteria: (a) Dec $>8^{\circ}$; (b) $|\mathrm{Gb}|>3^{\circ}$; (c) surface magnetic field strength, $B_{\text {surf }}>10^{10} \mathrm{G}$; (d) positional uncertainty within half of LOFAR's beam width at the upper edge of the HBA band (130", van Haarlem et al. 2013); and (e) not part of a globular cluster. For a more detailed discussion of the selection criteria, see the paper B16 noted above.

Ideally, the LBA extension of the HBA census would include all pulsars that had been detected with the high-band antennas except, perhaps, those pulsars with considerable scattering and which demonstrated no prospect for detecting very strong single pulses. In practice, when this project started, the HBA census was not yet processed or completed, and only preliminary detection estimates were available.

Originally, observations using the LBAs were meant to be conducted with an incoherent dedispersion scheme. Under this scheme, the observing band is split into many narrow channels and interstellar dispersion is only compensated for between channels but not within the channels themselves. The proposed source sample, therefore, only included pulsars with sufficiently small intra-channel smearing at $30 \mathrm{MHz}$, with the exact smearing threshold depending on the preliminary signalto-noise ratio $(\mathrm{S} / \mathrm{N})$ estimates from the HBA census, made without proper radio frequency interference (RFI) excision or updated ephemerides. We did not exclude sources with considerable scattering in the LBA band in the hope of detecting strong single pulses.

Before the start of observations with the LBAs, the incoherent dedispersion observing scheme was replaced with a coherent observing scheme, which made the intra-channel smearing criterion obsolete. However, the initial target list for the LBA follow-up remained unchanged. At present, with all HBA observations being processed and analysed (leading to substantial changes in some of the $\mathrm{S} / \mathrm{N}$ estimates), we can regard the LBA census source sample as being an arbitrary subsample of pulsars detected in the HBA census, with some preference towards closer and/or brighter sources (see Fig. 1).

\footnotetext{
1 http://www.epta.eu.org/epndb

2 http://www.atnf.csiro.au/people/pulsar/psrcat/ (Manchester et al. 2005).
}

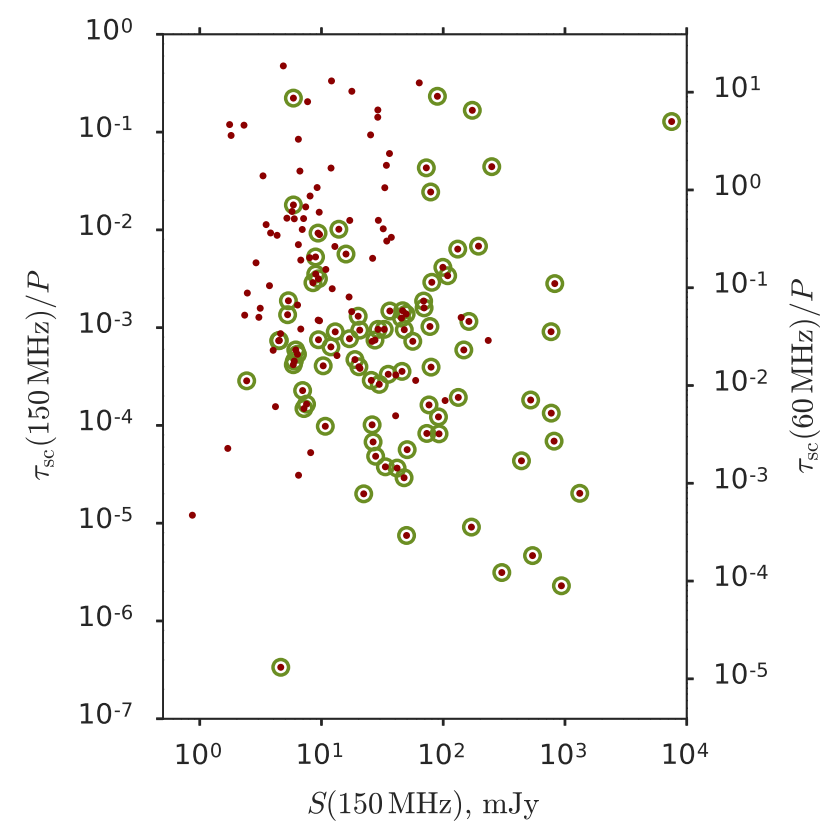

Fig. 1. Band-integrated fluxes and ratio of scattering time in the middle of HBA (left $y$-axis) and LBA (right $y$-axis) bands to the pulsar spin period for all sources detected in HBA census (red dots). Green circles mark the pulsars selected for the follow-up with LBAs. Scattering time is estimated with the Galactic electron density model from Yao et al. (2017) and scaled to respective frequencies with an exponent, $\alpha=-4.0$ in $\tau_{\mathrm{sc}} \sim v^{\alpha}$.

\section{Observations and data reduction}

Similarly to the HBA census, each pulsar was observed during one session for either at least 1000 rotational periods or at least $20 \mathrm{~min}$. The pulsars were observed between June 2014May 2015 using the LBAs of the LOFAR core stations in the frequency range of $30-89 \mathrm{MHz}$. In order to compensate for the refraction in the ionosphere, seven beams were formed around each source (beam 0 on the target and beams 1-6 in a hexagonal grid around beam 0 on the nominal position of the target) at a distance of about $210^{\prime \prime}$, approximately half of the telescope resolution at $60 \mathrm{MHz}$ (412.5", van Haarlem et al. 2013). The coordinates of the sources were taken from the ATNF pulsar catalogue or from the timing observations conducted with the Lovell telescope at Jodrell Bank and the 100-m Robert C. Byrd Green Bank Telescope.

For each beam, the coherently-summed complex-voltage signal from individual stations was coherently dedispersed. Raw data were stored in the LOFAR Long-Term Archive ${ }^{3}$. For a more detailed description of LOFAR and its pulsar observing modes, see van Haarlem et al. (2013) and Stappers et al. (2011).

Observations were pre-processed with the standard LOFAR pulsar pipeline (Stappers et al. 2011), which uses the PSRCHIVE software package (van Straten et al. 2010). Raw data were converted to full-Stokes samples which were recorded in PSRFITS format (Hotan et al. 2004), with time resolution of $5.12 \mu \mathrm{s}$ and 300 channels of $195 \mathrm{kHz}$. Folding produced 5-s sub-integrations with 1024 phase bins. In this paper, we focus solely on total intensity data. Table B.1 gives the basic observation summary for all pulsars in the LBA sample.

In most cases, the raw data were folded using the same ephemerides that were used for folding the HBA census data.

3 https://lta.lofar.eu/ 

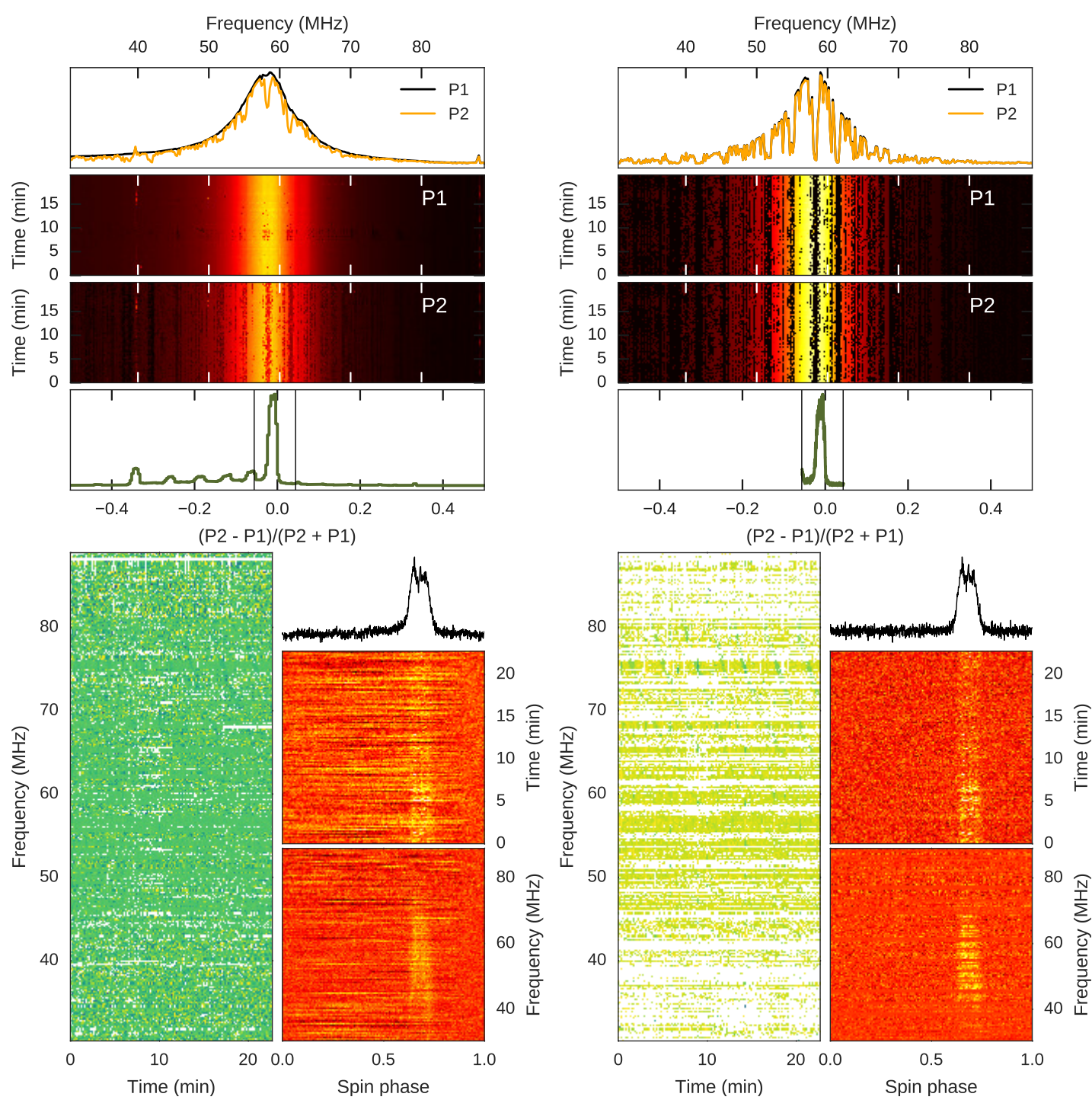

Fig. 2. Example of diagnostic plots without (left) and with (right) dropped packet cleaning applied for one observation of bright pulsar B0809+74. Upper row: statistics for two polarisations. Lower plots: dynamic and folded spectra, waterfall diagram, and the average profile.

An analysis of the HBA census data revealed that in many cases the DM as derived from higher-frequency observations was substantially different from the one obtained from census data. Thus, dedispersing and folding LBA data using incorrect DMs caused substantial pulse smearing within one frequency channel. To mitigate that effect, we rededispersed (coherently) and refolded 25 pulsars that were affected the most by using the DM value obtained in the HBA census. For the remaining pulsars, the smearing was less than one phase bin at $60 \mathrm{MHz}$ for the downsampled number of bins used in the analysis.

After the observations took place, we found that a substantial fraction of data packets were dropped, resulting in numerous data gaps ${ }^{4}$. These gaps appeared independently in two polarisations because of how the data is recorded to disk and led to significant decrease in overall $\mathrm{S} / \mathrm{N}$ (Fig. 2, left). In order to

\footnotetext{
4 The observations were carrying out during the time when new Cobalt correlator was put online. But, unfortunately, one of the network switches was misconfigured that resulted in somewhat lower network throughput for the used observing setup that was preliminary tested with the old $\mathrm{BG} / \mathrm{P}$ beamformer.
}

mitigate this adverse effect, we performed an additional step during the RFI cleaning procedure on all data archives. Working with 5-s, 300-channel archives with two polarisations $\left(P_{1}=X X^{*}\right.$ and $\left.P_{2}=Y Y^{*}\right)$, we computed the histogram of the relative signal strength difference, $d P=\left(P_{2}-P_{1}\right) /\left(P_{2}+P_{1}\right)$ for each 5-s/195$\mathrm{kHz}$ data cell. We then assigned zero weights to the cells with $d P$ deviating more than by 0.05 from the peak of the histogram (Fig. 2, right).

Since the bandpass in the LBA band is not uniform and has a large peak in sensitivity in the middle of the band, it is necessary to flatten the bandpass before cleaning RFI. Thus, we divided the dynamic spectrum by an "ideal bandpass" obtained from interpolating the median bandpass from all observations. To remove RFI from the flattened data, we used the clean. py tool from the CoastGuard package (Lazarus et al. 2016).

Archives that were automatically excised of RFI were also visually inspected for residual RFI. In many cases, the cleaning procedure was not entirely sufficient, resulting in some relatively faint RFI biasing the baseline estimates for flux calibration. For only three pulsars (namely, PSRs B0105+68, B0643+80, and B0656+14), the RFI prevented useful analysis so they were excluded from our sample. 
Overall, the fraction of band that has been zapped due to dropped packets or RFI is quite substantial, ranging from a few percent to almost the entire band (Table B.1). Zapped fraction varies considerably from beam to beam and is present in most observing runs, without showing a clear dependence on the observing date. While the data used here may not use LOFAR to its full capabilities, and ongoing and future low-frequency observations may reach higher $\mathrm{S} / \mathrm{N}$, the results we present here still provide useful information about the low-frequency end of the pulsar spectra (see Sect. 4.2).

\subsection{Detection and ephemerides update}

We adjusted the folding period $P$ and the intra-channel dispersive delay with the PSRCHIVE program pdmp, maximising the integrated $\mathrm{S} / \mathrm{N}$ of the frequency- and time-averaged profile. Initially, the entire band was used and the diagnostic output from pdmp was visually inspected for a pulsar-like signal. For those pulsars that were non-detected using this manner, or those with spectra that were not visually present across the whole band, we additionally zapped the edges of the band where the sensitivity is low and repeated the search for frequencies between 41 and $78 \mathrm{MHz}$. To facilitate visual inspection of the average profiles, we downsampled the initial number of phase bins by a factor of 2,4 or 8 .

It is worth mentioning that our DM measurements, which were based on maximising the $\mathrm{S} / \mathrm{N}$ of the frequency-integrated profile, did not take into account any profile evolution, which usually becomes rapid in the LBA band. Therefore, the reported $\mathrm{DM}$ values may be subject to a bias depending on the assumed profile evolution model.

Figure 3 shows the correlation between DM and the estimated scattering time over pulsar period for the detected and non-detected pulsars. The same information is also available in Table B.1. Our detections do not extend beyond a DM of $\sim 60 \mathrm{pc} \mathrm{cm}^{-3}$ and an estimated scattering time fraction of $\sim 20 \%$ of the pulse period.

Interestingly enough, one of the pulsars closest to Earth in our sample, namely, J1503+2111, was not detected. This pulsar had an ostensible error in DM measurements and the HBA census found it at DM $=3.260 \pm 0.004 \mathrm{pc} \mathrm{cm}^{-3}$ instead of the previously published $\mathrm{DM}=11.75 \pm 0.06 \mathrm{pc} \mathrm{cm}^{-3}$ (Champion et al. 2005). The pulsar was subsequently detected in HBAs with the LOFAR French station FR606 at the DM of the HBA census and this DM was used for folding in the current work. Since scattering is unlikely to be at play at this low DM, it is reasonable to assume that in our LBA observations that the pulsar has not been detected either because it is intermittent or because its flux density is too low. The upper limit on the band-integrated flux density is $\sim 35 \mathrm{mJy}$ (Table B.1), which is comparable to the predicted flux density from the HBA census $(\sim 20 \mathrm{mJy})$ so there is no clear indication of the spectral turnover. Note that both the upper limit and the predicted flux density value are subject to large, poorly-constrained uncertainties.

\subsection{Flux calibration}

The folded data files were calibrated the same way as in the HBA census (see B16 and Kondratiev et al. 2016 for more details). In short, we have established the flux density scale using the radiometer equation (Dicke 1946), which expresses the noise power through frequency-dependent antenna and sky temperatures, frequency- and direction-dependent telescope gain,

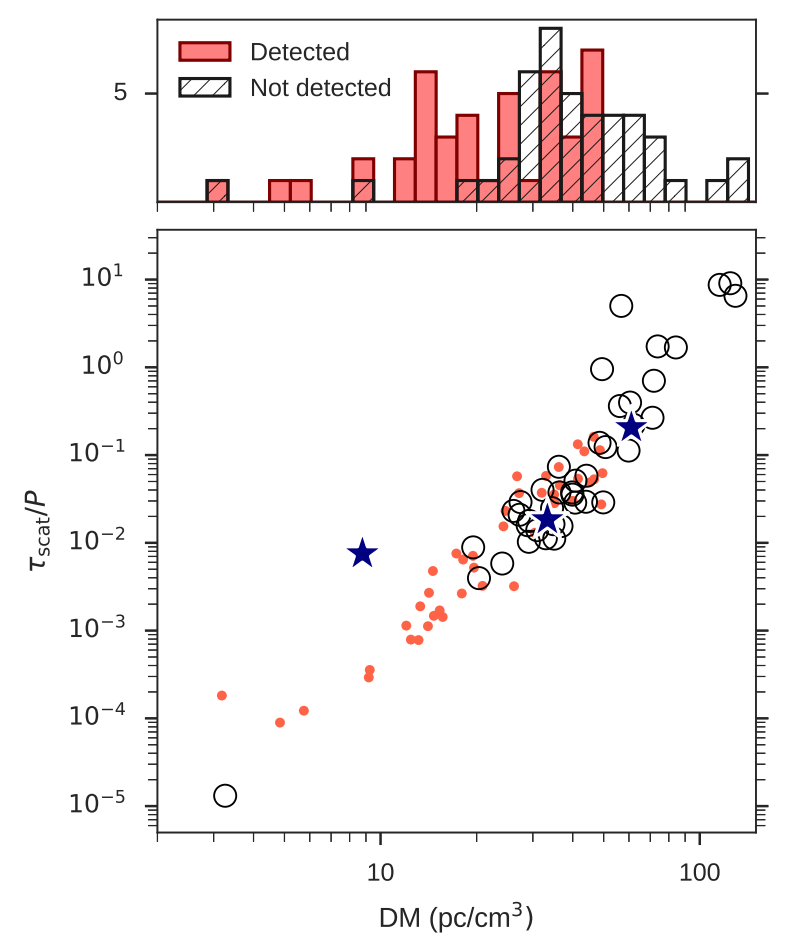

Fig. 3. Detected pulsars (red dots) and non-detected pulsars (black circles) versus DM and estimated scattering time at $60 \mathrm{MHz}$ divided by the pulsar period. Scattering times at $1 \mathrm{GHz}$ were taken from the Yao et al. (2017) electron density model and scaled to $60 \mathrm{MHz}$ with an exponent, $\alpha=-4.0$ in $\tau_{\mathrm{sc}} \sim v^{\alpha}$. Dark blue stars mark pulsars discarded due to an excess of RFI.

observing bandwidth, integration time, and the number of polarisations summed. The instrument temperature was derived from the measurements of Wijnholds \& van Cappellen (2011). The background sky temperature was scaled down to LBA frequencies from 408-MHz maps of Haslam et al. (1982) with the spectral index of -2.55 (Lawson et al. 1987). For the antenna gain, we used the Hamaker model of a station beam (Hamaker 2006) calculated using the mscorpol ${ }^{5}$ package by Tobia Carozzi. A coherence factor of 0.85 was used to scale the antenna gain with the actual number of stations involved in a given observation (Kondratiev et al. 2016).

For the Crab pulsar, the sky temperature was complemented with the contribution from the nebula. The latter was estimated using the relation $S_{\mathrm{Jy}} \approx 955 v_{\mathrm{GHz}}^{-0.27}$ (Bietenholz et al. 1997; Cordes et al. 2004). At $75 \mathrm{MHz}$, the solid angle occupied by the nebula (radius of $240^{\prime \prime}$, Bietenholz et al. 1997) is smaller than the full-width at the half-maximum of the LOFAR LBA beam (412.5" van Haarlem et al. 2013), thus the entire nebula is contributing to the system temperature. We must note that the Crab pulsar is scattered for more than a pulse period in the LBA band, thus the upper limit on the flux density is purely nominal, and it is, in fact, much smaller than the pulsar point source flux density measurements (B16). The on- and off-pulse windows for calculating the mean $\mathrm{S} / \mathrm{N}$ of the pulse profile were selected manually for each pulsar. Calibration was performed in each of 5-s subintegrations and 50 subbands of $11.7 \mathrm{MHz}$. Zero-weighted subbands and sub-integrations were excluded from the calculation of total bandwidth and observing time.

The nominal error on the flux density measurement, $\epsilon_{S \text { nom }}$, set by the noise in the off-pulse window does not fully reflect

5 https://github.com/2baOrNot2ba/mscorpol 
the true flux density measurement uncertainty since the latter is also influenced by the uncertainties in telescope parameters and scintillation. Since we did not observe any calibrator sources and were limited to only one session per pulsar, we cannot estimate those errors separately. The only way to verify our measurements is to compare the obtained flux density values with the ones from the literature. For those pulsars for which multiple spectra have been published (e.g. PSRs B0809+74, B1133+16, $\mathrm{B} 1508+55$, and others), the LBA measurements are consistent with the reported fluxes, which only vary by a factor of a few. More rigorous comparisons performed by B16 for the HBA data, based on multiple observing sessions and more numerous literature measurements in the HBA frequency range, suggest adopting $50 \%$ of measured flux density as the uncertainty on telescope parameters and scintillation. In this work, we extend this uncertainty estimate to the LBA frequency range, deferring a thorough study of telescope performance to a future work. For our observing setup and pulsar sample, the scintillation-induced flux modulation index, calculated with the basic theory of diffractive and refractive scintillation (Appendix A), is on the order of few percent for the majority of pulsars in the sample (but can be as large as $21 \%$, e.g. for the low-DM pulsar J1503+2111). The total flux density error was calculated by adding the nominal and the $50 \%$ errors in quadrature: $\epsilon_{S}=\sqrt{(0.5 S)^{2}+\epsilon_{S \text { nom }}^{2}}$.

The mean band-integrated flux densities and their respective uncertainties are listed in Table B.1. For non-detected pulsars we adopted three times the nominal error as the upper limit, although this does not take into account possible signal smearing due to scattering.

Our observing setup involved six beams in a circle surrounding the central beam formed towards the pulsars' coordinates. Interestingly, 19 out of 44 detected pulsars were detected with the highest $\mathrm{S} / \mathrm{N}$ in a side beam. This is indicative of refraction in the Earth's ionosphere due to differential total electron content (TEC) between the lines-of-sight of different LBA stations. We use the formula for the angular shift due to ionospheric refraction from Loi et al. (2015):

$|\Delta \theta|=\frac{40.3}{v^{2}}\left|\nabla_{\perp} \mathrm{TEC}\right|$

Here the numerical coefficient stems from the combination of fundamental physical constants, the angular shift $\Delta \theta$ is in radians, the transverse gradient of total electron content (along the line of sight in the ionosphere) $\nabla_{\perp}$ TEC is in electrons $\mathrm{m}^{-3}$, and $v$ is in Hz. For the rough estimate of possible values of TEC gradients, we used differences in TEC measured by the core HBA LOFAR stations in van Weeren et al. (2016). For $\delta$ TEC $10^{14} \mathrm{~m}^{-2}$ over the core size of $80 \mathrm{~km}, \nabla_{\perp}$ TEC is on the order of $10^{11} \mathrm{~m}^{-2}$. At the centre of LBA band $(v=60 \mathrm{MHz})$, this gives $|\Delta \theta| \sim 230^{\prime \prime}$, which is comparable to the beam separation. Smaller TEC differences, also reported by van Weeren et al. (2016), would result in smaller refraction angles.

Appendix B shows average profiles, band-integrated, or in 2-4 subbands in the case of strong pulsars or pulsars with an interesting profile evolution.

\section{Flux density spectra}

\subsection{Fitting}

For the pulsars that were detected, flux density values were combined with the published measurements (see Table B.1 for the full list of references) and these broadband spectra were fitted with a collection of power law (PL) functions. Similar to B16, a Bayesian fit was performed in $\lg S-\lg v$ space. Each $\lg S$ was modelled as a normally distributed random variable. The mean of the normal distribution was defined by the assumed PL dependence and the standard deviation reflected any kind of intrinsic variability or measurement uncertainty. See B16 for the remarks on PL applicability in general and the choice of a $\lg S$ model in particular.

Depending on the number of flux density measurements, we have approximated $\lg S_{\text {PL }}$ either as a single PL (hereafter "1PL"):

$\lg S_{1 \mathrm{PL}}=\alpha \lg \left(v / v_{0}\right)+\lg S_{0}$,

a broken PL with one break (2PL):

$\lg S_{2 \mathrm{PL}}= \begin{cases}\alpha_{\mathrm{lo}} \lg \left(v / v_{0}\right)+\lg S_{0}, & v<v_{\mathrm{br}} \\ \alpha_{\mathrm{hi}} \lg \left(v / v_{\mathrm{br}}\right)+\alpha_{\mathrm{lo}} \lg \left(v_{\mathrm{br}} / v_{0}\right)+\lg S_{0}, & v>v_{\mathrm{br}},\end{cases}$

or a broken PL with two breaks (3PL):

$\lg S_{3 \mathrm{PL}}= \begin{cases}\alpha_{\mathrm{lo}} \lg \left(v / v_{0}\right)+\lg S_{0}, & v<v_{\mathrm{br}}^{\mathrm{lo}} \\ \alpha_{\text {mid }} \lg \left(v / v_{\mathrm{br}}^{\mathrm{lo}}\right)+\alpha_{\mathrm{lo}} \lg \left(v_{\mathrm{br}}^{\mathrm{lo}} / v_{0}\right)+\lg S_{0}, & v_{\mathrm{br}}^{\mathrm{lo}}<v<v_{\mathrm{br}}^{\mathrm{hi}} . \\ \alpha_{\mathrm{hi}} \lg \left(v / \nu_{\mathrm{br}}^{\mathrm{hi}}\right)+\alpha_{\text {mid }} \lg \left(v_{\mathrm{br}}^{\mathrm{hi}} / \nu_{0}\right)+\lg S_{0}, & v>v_{\mathrm{br}}^{\mathrm{hi}}\end{cases}$

The reference frequency $v_{0}$ was taken to be close to the geometric average of the minimum and maximum frequencies in the spectrum.

As in B16, for the small number of flux density measurements (treating all measurements within $10 \%$ in frequency as a single group), we fixed the uncertainty at the level defined by the reported errors. For a larger number of frequency groups, an additional fit parameter was introduced, the unknown error $\sigma_{\lg S}^{\mathrm{unkn}}$. A single error per source was fitted, representing intrinsic variability, or any kind of unaccounted propagation or instrumental error. The total flux density uncertainty of any $\lg S$ was then taken as the known and unknown errors added in quadrature.

The posterior distribution of $\sigma_{\lg S}^{\mathrm{unkn}}$ was used to discriminate between models. A result of 1PL was taken as a null hypothesis and rejected in favour of 2PL or 3PL if the latter was shown to give a statistically smaller $\sigma_{\lg S}^{\mathrm{unk}}$ (for details, see B16). If no $\sigma_{\lg S}^{\text {unkn }}$ was fitted, we adopted 1PL as the single model. In a few cases where the data showed a hint of a spectral break but no $\sigma_{\lg S}^{\mathrm{unkn}}$ was fitted, we fitted 2PL with the break frequency fixed at the frequency of the largest flux density measurement. For such pulsars, we give both 1PL and 2PL values of the fitted parameters. Upper limits on flux densities were not taken into account while fitting.

\subsection{Results}

Seventeen out of the 43 detected pulsars did not have published flux density measurements in the LBA frequency range. Some of them do not show signs of scattering, which indicates an opportunity to study these pulsars at even lower frequencies: for example, PSRs B0011+47, B0226+70, and B2022+50 did not exhibit any sign of low-frequency turnover or apparent scattering down to $40 \mathrm{MHz}$ (see Fig. C.1).

Overall, our new spectral indices are very similar to those published in B16. For pulsars with relatively well-measured spectra, the LBA flux densities agree reasonably well with previous measurements in this frequency range. In some cases (e.g. PSRs B0450+55, B0655+64, and B2217+47), LBA flux densities are lower by a factor of a few with respect to the 
measurements of Stovall et al. (2015). However, this is not the case for all LBA census pulsars that overlap with their sample (e.g. PSR B1929+21) and there is at least one example where fluxes from Stovall et al. (2015) are much higher than the bulk of other literature measurements in the same frequency region (PSR B1133+16).

For pulsars with fewer spectral points and no previous measurements below $100 \mathrm{MHz}$, LBA fluxes did not span enough of the frequency range to have a large influence on the spectral index. For some sources, the $\mathrm{S} / \mathrm{N}$ was sufficient to break the LBA band into two or more subbands and the flux density values hint to a possible spectral break (e.g. PSR J0611+30), however large errors and close proximity in frequency between the new data points make it so that the break is not statistically significant.

Some of the sources had a different number of PL breaks than those noted in B16 (e.g. PSRs B0450+55, B1133+16, $\mathrm{B} 1811+40$, B2217+47). This mostly stems from the influence of separate flux measurements on the fitted $\sigma_{\lg S}^{\mathrm{unkn}}$ since we fitted only one unknown error per source. Sometimes the lowfrequency (or even high-frequency) breaks were substantially different than those of B16 (e.g. PSRs B0823+26, B1530+27, and $\mathrm{B} 1737+13)$.

It is worth mentioning that in parallel to this study, a similar census of the pulsar population visible below $100 \mathrm{MHz}$ was undertaken by the LOFAR international station FR606 (Bondonneau et al. 2020). They observed a similar sample of pulsars (103 compared to the 88 pulsars of the present study). The reduced collecting area $(\sim 10 \%)$ was compensated by long integrations (on average $3 \mathrm{~h}$ per target). With this method, the authors detected 64 pulsars, compared with the 43 pulsars of the present study. For a detailed comparison of the results, we refer the reader to Bondonneau et al. (2020).

\section{Discussion}

Despite its limitations, the LBA extension of the HBA census provides useful information for identifying more than a dozen pulsars suitable for the subsequent follow-up at the lowest frequencies observable from Earth. We provide reference average profiles and fluxes for the 43 pulsars detected, 17 of them having no previously published flux densities at these frequencies.

Overall, the main concerns raised in B16 remain standing: despite being one of the basic characteristics of pulsars, their spectra remain, to a large extent, poorly constrained due to the lack of robust, systematic multifrequency measurements. The situation is even worse in the low-frequency range, where a spectral break is widely expected.

Proper quantification of the low-frequency spectral break is essential for studying the emission mechanism and propagation of radio waves in the magnetosphere. The existence of a low-frequency turnover has been previously attributed to a number of physical processes, for example, synchrotron selfabsorption (Sieber 1973), refraction of the ordinary radio wave mode (Beskin 2018), free-free absorption (Malov 1979), or stimulated scattering (Lyubarskii \& Petrova 1996). In particular, Beskin (2018) proposes a clear dependence of the turnover frequency on the pulsar spin period, something that may be readily verified using the future observations. However, such a study would require a large number of well-measured spectra from pulsars from substantially different periods. Furthermore, the influence of the ISM on the observed flux densities (for example, a decrease in apparent pulsed flux density due to scattering) should be carefully accounted for in, for exam- ple, measuring continuum flux densities at low radio frequencies (Shimwell et al. 2019).

Future observations of pulsars below $100 \mathrm{MHz}$ can provide more robust flux density measurements and better constrain spectral break(s). This will be achieved, in particular, by the NenuFAR radio telescope (Zarka et al. 2012, 2014, 2015) and its pulsar instrumentation LUPPI (Bondonneau et al. 2019), which we are currently using to conduct a systematic census of the pulsar population (Bondonneau et al. 2020). Due to its sensitivity, its constant antenna response across its frequency band $(10-85 \mathrm{MHz})$ and long integrations, it is expected that NenuFAR will be capable of detecting a much larger number of pulsars compared to the LBA census and its companion study with FR606.

Acknowledgements. This works makes extensive use of matplotlib (http: //matplotlib.org/) (Hunter 2007), seaborn (http://stanford.edu/ mwaskom/software/seaborn/) Python plotting libraries and NASA's Astrophysics Data System. This paper is based on data obtained with the International LOFAR Telescope (ILT) under project code LC2_025. LOFAR (van Haarlem et al. 2013) is the Low Frequency Array designed and constructed by ASTRON. It has observing, data processing, and data storage facilities in several countries, that are owned by various parties (each with their own funding sources), and that are collectively operated by the ILT foundation under a joint scientific policy. The ILT resources have benefited from the following recent major funding sources: CNRS-INSU, Observatoire de Paris and Université d'Orléans, France; BMBF, MIWF-NRW, MPG, Germany; Science Foundation Ireland (SFI), Department of Business, Enterprise and Innovation (DBEI), Ireland; NWO, The Netherlands; The Science and Technology Facilities Council, UK.

\section{References}

Barr, E. D., Champion, D. J., Kramer, M., et al. 2013, MNRAS, 435, 2234 Bartel, N., Sieber, W., \& Wielebinski, R. 1978, A\&A, 68, 361 Beskin, V. S. 2018, Phys. Usp., 61, 353

Bhat, N. D. R., Rao, A. P., \& Gupta, Y. 1999, ApJS, 121, 483

Bietenholz, M. F., Kassim, N., Frail, D. A., et al. 1997, ApJ, 490, 291

Bilous, A. V., Kondratiev, V. I., Kramer, M., et al. 2016, A\&A, 591, A134

Bondonneau, L., Grießmeier, J., Theureau, G., Cognard, I., \& the NenuFAR-

France Collaboration 2019, Journées scientifiques 2019 d'URSI-France, 1

Bondonneau, L., Grießmeier, J.-M., Theureau, G., et al. 2020, A\&A, 635, A76

Boyles, J., Lynch, R. S., Ransom, S. M., et al. 2013, ApJ, 763, 80

Bridle, A. H. 1970, Nature, 225, 1035

Camilo, F., \& Nice, D. J. 1995, ApJ, 445, 756

Camilo, F., Nice, D. J., Shrauner, J. A., \& Taylor, J. H. 1996, ApJ, 469, 819

Champion, D. J., Lorimer, D. R., McLaughlin, M. A., et al. 2005, MNRAS, 363, 929

Cordes, J. M., Bhat, N. D. R., Hankins, T. H., McLaughlin, M. A., \& Kern, J. 2004, ApJ, 612, 375

Damashek, M., Taylor, J. H., \& Hulse, R. A. 1978, ApJ, 225, L31

Davies, J. G., Lyne, A. G., \& Seiradakis, J. H. 1977, MNRAS, 179, 635

Dembska, M., Kijak, J., Jessner, A., et al. 2014, MNRAS, 445, 3105

Deshpande, A. A., \& Radhakrishnan, V. 1992, JApA, 13, 151

Dewey, R. J., Taylor, J. H., Weisberg, J. M., \& Stokes, G. H. 1985, ApJ, 294, L25

Dicke, R. H. 1946, Rev. Sci. Instrum., 17, 268

Downs, G. S. 1979, ApJS, 40, 365

Downs, G. S., Reichley, P. E., \& Morris, G. A. 1973, ApJ, 181, L143

Fomalont, E. B., Goss, W. M., Lyne, A. G., Manchester, R. N., \& Justtanont, K. 1992, MNRAS, 258, 497

Gould, D. M.1994, PhD Thesis, Univ. of Manchester

Hamaker, J. P. 2006, A\&A, 456, 395

Han, J. L., Demorest, P. B., van Straten, W., \& Lyne, A. G. 2009, ApJS, 181, 557 Haslam, C. G. T., Salter, C. J., Stoffel, H., \& Wilson, W. E. 1982, A\&AS, 47, 1 Hewish, A., Bell, S. J., Pilkington, J. D. H., Scott, P. F., \& Collins, R. A. 1968, Nature, 217, 709

Hobbs, G., Faulkner, A., Stairs, I. H., et al. 2004, MNRAS, 352, 1439

Hotan, A. W., van Straten, W., \& Manchester, R. N. 2004, Proc. Astron. Soc., 21,302

Hunter, J. D. 2007, Comput. Sci. Eng., 9, 90

Izvekova, V. A., Kuz'min, A. D., Malofeev, V. M., \& Shitov, Y. P. 1979, Sov. Astron., 23, 179 
Izvekova, V. A., Kuzmin, A. D., Malofeev, V. M., \& Shitov, I. P. 1981, Ap\&SS, 78,45

Jankowski, F., van Straten, W., Keane, E. F., et al. 2018, MNRAS, 473, 4436

Johnston, S., Karastergiou, A., \& Willett, K. 2006, MNRAS, 369, 1916

Kaplan, D. L., Condon, J. J., Arzoumanian, Z., \& Cordes, J. M. 1998, ApJS, 119 75

Karuppusamy, R., Stappers, B. W., \& Serylak, M. 2011, A\&A, 525, A55

Kijak, J., Kramer, M., Wielebinski, R., \& Jessner, A. 1998, A\&AS, 127, 153

Kijak, J., Gupta, Y., \& Krzeszowski, K. 2007, A\&A, 462, 699

Kondratiev, V. I., Verbiest, J. P. W., Hessels, J. W. T., et al. 2016, A\&A, 585, A128

Kramer, M., Xilouris, K. M., Jessner, A., Wielebinski, R., \& Timofeev, M. 1996 A\&A, 306, 867

Kramer, M., Jessner, A., Doroshenko, O., \& Wielebinski, R. 1997, ApJ, 488, 364

Krzeszowski, K., Maron, O., Słowikowska, A., Dyks, J., \& Jessner, A. 2014 MNRAS, 440, 457

Kuz'min, A. D., Malofeev, V. M., Shitov, Y. P., et al. 1978, MNRAS, 185, 441

Kuzmin, A. D., Malofeev, V. M., Izvekova, V. A., Sieber, W., \& Wielebinski, R. 1986, A\&A, 161, 183

Lane, W. M., Cotton, W. D., van Velzen, S., et al. 2014, MNRAS, 440, 327

Lawson, K. D., Mayer, C. J., Osborne, J. L., \& Parkinson, M. L. 1987, MNRAS, 225, 307

Lazarus, P., Karuppusamy, R., Graikou, E., et al. 2016, MNRAS, 458, 868

Lewandowski, W., Wolszczan, A., Feiler, G., Konacki, M., \& Sołtysiński, T. 2004, ApJ, 600, 905

Löhmer, O., Jessner, A., Kramer, M., Wielebinski, R., \& Maron, O. 2008, A\&A, 480,623

Loi, S. T., Murphy, T., Bell, M. E., et al. 2015, MNRAS, 453, 2731

Lommen, A. N., Zepka, A., Backer, D. C., et al. 2000, ApJ, 545, 1007

Lorimer, D. R., \& Kramer, M. 2005, Handbook of Pulsar Astronomy (Cambridge: Cambridge University Press)

Lorimer, D. R., Yates, J. A., Lyne, A. G., \& Gould, D. M. 1995, MNRAS, 273 411

Lorimer, D. R., Xilouris, K. M., Fruchter, A. S., et al. 2005, MNRAS, 359, 1524

Lyubarskii, Y. E., \& Petrova, S. A. 1996, Astron. Lett., 22, 399

Malofeev, V. M. 1993, Astron. Lett., 19, 138

Malofeev, V. M.1999, Katalog radiospektrov pulsarov [in Russian], Pushchino Radio Astronomy Observatory

Malofeev, V. M., \& Malov, I. F. 1980, Sov. Astron., 24, 54

Malofeev, V. M., Malov, O. I., \& Shchegoleva, N. V. 2000, Astron. Rep., 44, 436

Malov, I. F. 1979, Sov. Astron., 23, 205

Manchester, R. N. 1971, ApJ, 163, L61

Manchester, R. N., \& Taylor, J. H. 1981, AJ, 86, 1953

Manchester, R. N., Lyne, A. G., Taylor, J. H., et al. 1978, MNRAS, 185, 409

Manchester, R. N., Hobbs, G. B., Teoh, A., \& Hobbs, M. 2005, AJ, 129, 1993

Maron, O., Kijak, J., Kramer, M., \& Wielebinski, R. 2000, A\&AS, 147, 195
Moffett, D. A., \& Hankins, T. H. 1999, ApJ, 522, 1046

Morris, D., Graham, D. A., Sieber, W., Bartel, N., \& Thomasson, P. 1981, A\&AS 46, 421

Murphy, T., Kaplan, D. L., Bell, M. E., et al. 2017, PASA, 34, e020

Navarro, J., Anderson, S. B., \& Freire, P. C. 2003, ApJ, 594, 943

Rankin, J. M., \& Benson, J. M. 1981, AJ, 86, 418

Rankin, J. M., Comella, J. M., Craft, Jr., H. D., et al. 1970, ApJ, 162, 707

Ray, P. S., Thorsett, S. E., Jenet, F. A., et al. 1996, ApJ, 470, 1103

Sayer, R. W., Nice, D. J., \& Taylor, J. H. 1997, ApJ, 474, 426

Seiradakis, J. H., Gil, J. A., Graham, D. A., et al. 1995, A\&AS, 111, 205

Shimwell, T. W., Tasse, C., Hardcastle, M. J., et al. 2019, A\&A, 622, A1

Shrauner, J. A., Taylor, J. H., \& Woan, G. 1998, ApJ, 509, 785

Sieber, W. 1973, A\&A, 28, 237

Sieber, W., \& Wielebinski, R. 1987, A\&A, 177, 342

Slee, O. B., Alurkar, S. K., \& Bobra, A. D. 1986, Aust. J. Phys., 39, 103

Stappers, B. W., Hessels, J. W. T., Alexov, A., et al. 2011, A\&A, 530, A80

Stinebring, D. R., \& Condon, J. J. 1990, ApJ, 352, 207

Stokes, G. H., Segelstein, D. J., Taylor, J. H., \& Dewey, R. J. 1986, ApJ, 311, 694

Stovall, K., Ray, P. S., Blythe, J., et al. 2015, ApJ, 808, 156

Taylor, J. H., Manchester, R. N., \& Lyne, A. G. 2000, VizieR Online Data Catalog: VII/189

Thorsett, S. E., Deich, W. T. S., Kulkarni, S. R., Navarro, J., \& Vasisht, G. 1993 ApJ, 416, 182

van Haarlem, M. P., Wise, M. W., Gunst, A. W., et al. 2013, A\&A, 556, A2

van Straten, W., Manchester, R. N., Johnston, S., \& Reynolds, J. E. 2010, PASA, 27,104

van Weeren, R. J., Williams, W. L., Hardcastle, M. J., et al. 2016, ApJS, 223, 2

Vivekanand, M., Mohanty, D. K., \& Salter, C. J. 1983, MNRAS, 204, 81P

Wang, N., Manchester, R. N., Johnston, S., et al. 2005, MNRAS, 358, 270

Wielebinski, R., Jessner, A., Kramer, M., \& Gil, J. A. 1993, A\&A, 272, L13

Wijnholds, S. J., \& van Cappellen, W. A. 2011, IEEE Trans. Antennas Propag., 59,1981

Xue, M., Bhat, N. D. R., Tremblay, S. E., et al. 2017, PASA, 34, e070

Yao, J. M., Manchester, R. N., \& Wang, N. 2017, ApJ, 835, 29

Zakharenko, V. V., Vasylieva, I. Y., Konovalenko, A. A., et al. 2013, MNRAS, 431, 3624

Zarka, P., Girard, J. N., Tagger, M., Denis, L., \& the LSS Team 2012, in SF2A2012: Semaine de l'Astrophysique Francaise, 687

Zarka, P., Tagger, M., Denis, L., et al. 2014, NenuFAR: Instrument Description and Science Case, Tech. rep., https://www.researchgate.net/ publication/308806477_NenUFAR_Instrument_description_and_ science_case

Zarka, P., Tagger, M., Denis, L., et al. 2015, International Conference on Antenna Theory and Technique, 13

Zhao, R.-S., Wu, X.-J., Yan, Z., et al. 2017, ApJ, 845, 156 


\section{Appendix A: Scintillation}

The variation of observed flux due to scintillation on the inhomogeneities in the ISM was estimated with a simple thinscreen Kolmogorov model (see Lorimer \& Kramer 2005, for review). The scintillation bandwidth was taken to be $\Delta f=$ $1.16 /\left(2 \pi \tau_{\text {scat }}\right) \times(60 \mathrm{MHz} / 1 \mathrm{GHz})^{4.0}$, where $\tau_{\text {scat }}$ is scattering time at $1 \mathrm{GHz}$ from Yao et al. (2017). For all census pulsars, the scintillation bandwidth $\Delta f$ was smaller than a few $\mathrm{kHz}$, satisfying the conditions of strong scintillation regime $(\sqrt{f / \Delta f}>1)$.

Diffractive interstellar scintillation (DISS) did not have a large impact on the flux variation since many scintles were averaged in the frequency domain, resulting in modulation index $m_{\text {DISS }}$ (rms of the flux density divided by its mean value) was on the order of a percent or less. The refractive scintillation (RISS) was much stronger with typical $m_{\text {RISS }} \approx 0.05-0.1$. Table B.1 lists the expected values of total modulation index.

\section{Appendix B: Tables}

Table B.1 summarises the observations. The columns indicate: pulsar name; approximate spin period (s); observing epoch
(MJD); duration of an observing session ( $\mathrm{min}$ ); frequency range $(\mathrm{MHz}$ ); best beam (usually the one with highest $\mathrm{S} / \mathrm{N}$ ); fraction of zapped data in the dynamic spectrum for the least and the most affected beams; peak $\mathrm{S} / \mathrm{N}$ of the average profile; measured census DM; expected Yao et al. (2017) scattering time at $60 \mathrm{MHz}$ divided by pulsar period; expected modulation index due to scintillation in the ISM; and mean flux density within specified frequency range (upper limit for the non-detected pulsars), along with the literature references to previous flux density measurements. The values in parentheses indicate the errors on the last two significant digits.

Tables B.2-B. 4 contain best-fitted parameters for the pulsars with the spectra modelled with a single PL, a PL with one break, and a PL with two breaks, respectively. The columns include pulsar name; spectral frequency span $(\mathrm{MHz})$; number of data points in spectrum, $N_{p}$; the reference frequency, $v_{0}(\mathrm{MHz})$; flux density at the reference frequency, $S_{0}$ (mJy); spectral index (or indices in case of broken PLs), $\alpha$; and fitted flux density scatter, $\sigma_{\lg S}^{\text {unkn }}$, if applicable (see Sect. 4.1). Tables B.3 and B.4 also include break frequency, $v_{\mathrm{br}}(\mathrm{MHz})$, together with its $68 \%$ uncertainty range. 
Table B.1. Observation summary, DM, and flux density measurements.

\begin{tabular}{|c|c|c|c|c|c|c|c|c|c|c|c|c|}
\hline PSR & $\begin{array}{l}\text { Period } \\
P \\
(\mathrm{~s}) \\
\end{array}$ & $\begin{array}{l}\text { Observing } \\
\text { epoch } \\
\text { (MJD) }\end{array}$ & $\begin{array}{l}\text { Obs. } \\
\text { time } \\
(\mathrm{min})\end{array}$ & $\begin{array}{l}\text { Freq. } \\
\text { range } \\
(\mathrm{MHz})\end{array}$ & $\begin{array}{l}\text { Best } \\
\text { beam }\end{array}$ & $\begin{array}{l}\text { Zapped } \\
\text { fraction }\end{array}$ & $\begin{array}{l}\text { Peak } \\
S / N\end{array}$ & $\begin{array}{l}\mathrm{DM}_{\mathrm{cen}} \\
\left(\mathrm{pc} \mathrm{cm}^{-3}\right)\end{array}$ & $\begin{array}{l}\text { Expected } \\
\tau_{\text {scat }} / P\end{array}$ & $\begin{array}{l}\text { Exp. } \\
\text { mod. } \\
\text { index }\end{array}$ & $\begin{array}{l}\text { Mean } \\
\text { flux } \\
(\mathrm{mJy})\end{array}$ & $\begin{array}{l}\text { Literature flux } \\
\text { references }\end{array}$ \\
\hline B $0011+47$ & 1.241 & 56949.82 & 21 & $42.12-76.15$ & 0 & $0.21-0.43$ & 6 & $30.3048(65)$ & $1 \mathrm{e}-02$ & 0.08 & $45 \pm 25$ & $3,15,19,22,25,38,42,46,57$ \\
\hline B $0045+33$ & 1.217 & 57112.46 & 21 & $42.35-77.09$ & & $0.45-0.55$ & & $\ldots$ & $4 e-02$ & 0.06 & $<56.0$ & $3,16,38,46,49,57,64$ \\
\hline B $0052+51$ & 2.115 & 114.46 & 36 & $42.10-77.23$ & & $0.16-0.17$ & & & $3 e-02$ & 0.06 & $<22.6$ & $3,16,19,25,38,46,49$ \\
\hline B $0053+47$ & 0.472 & 57027.69 & 20 & $30.37-77.24$ & 5 & $0.05-0.06$ & 15 & $18.0954(10)$ & $6 e-03$ & 0.10 & $110 \pm 60$ & $3,16,28,38,49,64,69$ \\
\hline B $0105+68^{(\sharp)}$ & 1.071 & 57148.40 & 20 & $42.05-77.24$ & $\ldots$ & $0.81-0.85$ & $\ldots$ & $\ldots$ & $2 \mathrm{e}-01$ & 0.05 & $<140.7$ & $3,16,38,49,57,64$ \\
\hline B $0114+58$ & 0.101 & 57112.48 & 20 & $42.41-77.19$ & $\ldots$ & $0.48-0.63$ & $\ldots$ & $\ldots$ & $1 \mathrm{e}+00$ & 0.06 & $<99.8$ & $3,19,35,38,49,60$ \\
\hline $\mathrm{J} 0137+1654$ & 0.415 & 56827.22 & 20 & $42.15-77.29$ & 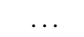 & $0.17-0.35$ & $\ldots$ & $\ldots$ & $2 e-02$ & 0.08 & $<42.8$ & $3,37,69$ \\
\hline B0136+57 & 0.272 & 56827.28 & 20 & $42.15-77.19$ & $\ldots$ & $0.26-0.45$ & $\ldots$ & $\ldots$ & $2 \mathrm{e}+00$ & 0.04 & $<47.8$ & $3,15,22,25,38,46,49,56$ \\
\hline B0153+39 & 1.811 & 56827.24 & 31 & $42.14-77.13$ & & $0.32-0.59$ & & & $1 e-01$ & 0.05 & $<52.6$ & $3,16,19,38,49,64$ \\
\hline B $0226+70$ & 1.467 & 56827.26 & 25 & $42.26-77.05$ & 0 & $0.22-0.36$ & 7 & $46.7394(66)$ & $5 e-02$ & 0.06 & $49 \pm 29$ & $3,16,38,42,46,49$ \\
\hline В $0301+19$ & 1.388 & 56975.00 & 24 & $42.13-77.13$ & 0 & $0.28-0.57$ & 10 & $15.6568(99)$ & $1 \mathrm{e}-03$ & 0.11 & $61 \pm 33$ & $\begin{array}{l}3,17,21,22,25,28,38,42,46 \\
47,48,49,56,57,59,69\end{array}$ \\
\hline В $0320+39$ & 3.032 & 56903.21 & 65 & $42.08-77.29$ & 3 & $0.38-0.41$ & 29 & $26.1698(20)$ & $3 e-03$ & 0.08 & $76 \pm 39$ & $\begin{array}{l}3,15,17,22,28,29,38,42,46, \\
57,59,69\end{array}$ \\
\hline $\mathrm{J} 0324+5239$ & 0.337 & 56903.25 & 20 & $42.16-77.18$ & $\ldots$ & $0.28-0.54$ & $\ldots$ & $\ldots$ & $9 \mathrm{e}+00$ & 0.03 & $<70.5$ & 2,3 \\
\hline B $0410+69$ & 0.391 & 56975 & 20 & $42.18-$ & & & & & & & & 3,16 \\
\hline $\mathrm{J} 0417+35$ & 0.654 & 56975 & 2 & $42.08-77.26$ & & $0.21-0.52$ & & & 01 & .06 & $<38.0$ & $3,9,49$ \\
\hline B $0450+55$ & 0.341 & 56903.26 & 20 & $42.23-76.93$ & 0 & $0.31-0.53$ & 12 & $14.590(77)$ & $5 e-03$ & 0.11 & $110 \pm 60$ & $\begin{array}{l}3,25,38,46,49,50,57,59,61 \text {, } \\
69\end{array}$ \\
\hline B $0531+21$ & 0.034 & 56903.27 & 20 & $41.89-77.28$ & $\ldots$ & $0.45-0.53$ & $\ldots$ & $\ldots$ & $5 e+00$ & 0.05 & $<70.6$ & $\begin{array}{l}1,3,28,38,40,42,44,48,49, \\
50,53,57\end{array}$ \\
\hline $\mathrm{J} 0611+30$ & 1.412 & 10 & 24 & 42.1 & 0 & 49 & 8 & 45.2 & $5 e-02$ & 0.06 & $89 \pm 46$ & 3,9 \\
\hline B $0609+37$ & 0.298 & 56975.07 & 20 & 42.10 & 4 & 41 & 6 & 27.17 & & 0.0 & $46 \pm 25$ & $3,28,38$ \\
\hline B $0626+24$ & 0.476 & 56903.29 & 20 & $42.04-77.24$ & $\ldots$ & $0.56-0.64$ & $\ldots$ & $\ldots$ & $2 \mathrm{e}+00$ & 0.04 & $<51.5$ & $\begin{array}{l}3,15,17,25,28,38,46,49,50, \\
57\end{array}$ \\
\hline B $0643+80^{(\sharp)}$ & 1.215 & 56903.34 & 21 & $42.17-77.09$ & & $0.68-0.79$ & & & $2 \mathrm{e}-02$ & 0.07 & $<66.8$ & $3,28,38,42,49,57$ \\
\hline B0655+64 & 0.196 & 569 & 20 & 42.29 & 0 & $0.60-0.67$ & 14 & $8.7739(19)$ & $2 \mathrm{e}-03$ & 14 & $86 \pm 49$ & $8,41,57,59,61,69$ \\
\hline B065 & 0.385 & 56903.33 & 20 & $42.06-77.06$ & $\ldots$ & $0.82-0.98$ & $\ldots$ & $\ldots$ & $4 e-03$ & 0.11 & $<94.8$ & $\begin{array}{l}3,22,23,24,38,46,47,49,57, \\
65,69,70\end{array}$ \\
\hline B $0751+32$ & 1.442 & & 25 & 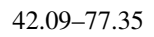 & 4 & 0 & 4 & 39.8 & & 0.06 & 21 & $3,15,22,28,38,49,57$ \\
\hline B0809 & 1.292 & 56903.37 & 22 & $30.64-77.17$ & 3 & $0.55-0.68$ & 34 & $5.7707(84)$ & $1 e-04$ & 0.17 & $1400 \pm 700$ & $\begin{array}{l}3,6,13,21,22,25,28,29,31, \\
34,38,42,43,46,48,49,50, \\
57,61,69\end{array}$ \\
\hline B $0823+26$ & 0.531 & 56975.12 & 20 & $30.37-77.19$ & 4 & $0.16-0.44$ & 107 & $19.4763(35)$ & $7 e-03$ & 0.10 & $700 \pm 350$ & $\begin{array}{l}3,5,6,10,14,18,21,22,25, \\
29,33,35,37,38,42,43,45 \\
46,48,49,50,57,59,61,66, \\
67,69\end{array}$ \\
\hline $\mathrm{B} 0841+80$ & 1.602 & & $2 \tau$ & & & & & & & & $<24.5$ & 3 , \\
\hline B091? & 1.568 & 56 & 27 & 34 & 0 & 51 & 13 & 13.15 & & & $41 \pm 22$ & 4,69 \\
\hline B0940+16 & 1.087 & 56903.40 & 20 & $42.01-77.19$ & $\ldots$ & $0.64-0.74$ & $\ldots$ & $\ldots$ & $4 e-03$ & 0.10 & $<61.2$ & $\begin{array}{l}3,17,22,24,37,38,47,57,65 \text {, } \\
69\end{array}$ \\
\hline $\mathrm{J} 0943+2$ & 0 & & 2 & & & & & & & & $<39.2$ & $3,49,63$ \\
\hline B09 & 1.098 & 56826.7 & 20 & $30.40-77.17$ & 0 & $0.16-0.36$ & 50 & $15.3585(72)$ & $2 \epsilon$ & 0.11 & $400 \pm 200$ & $\begin{array}{l}3,13,22,38,42,48,49,50,52, \\
57,61,69\end{array}$ \\
\hline J0947+27 & 0.851 & & 20 & 42 & & & & & & 0. & $<19.7$ & $3,54,69$ \\
\hline B $1112+50$ & 1.656 & 57028.15 & 28 & $30.37-77.24$ & 0 & $0.07-0.10$ & 26 & 9.1 & $3 e$ & 0.14 & $3 \pm 22$ & $\begin{array}{l}3,21,22,32,38,42,43,46,48 \\
49,50,57,59,69\end{array}$ \\
\hline B1133+16 & 1.188 & 56826.74 & 20 & $30.39-77.21$ & 4 & $0.17-0.27$ & 135 & $4.8407(78)$ & $9 e-05$ & 0.18 & \pm 440 & $\begin{array}{l}3,5,6,10,13,14,18,21,22, \\
23,24,25,29,31,30,32,33 \\
34,35,38,42,43,46,47,48 \\
49,50,57,59,61,62,66,68 \\
69,70\end{array}$ \\
\hline & & & 2 & & 4 & & 18 & & & & & $3,49,54,69$ \\
\hline B $1237+25$ & 1.383 & 56826.76 & 24 & $42.11-77.25$ & 4 & $0.08-0.12$ & 61 & $9.2716(90)$ & $4 e-04$ & 0.14 & $150 \pm 80$ & $\begin{array}{l}3,5,6,10,17,18,21,22,25, \\
29,31,38,42,43,46,48,49, \\
50,57,59,61,62,69,70\end{array}$ \\
\hline $\mathrm{J} 1313+0931$ & 0.849 & 56826.79 & 20 & $42.25-76.00$ & 4 & $0.19-0.39$ & 6 & $12.0406(15)$ & $1 \mathrm{e}-03$ & 0.12 & $24 \pm 19$ & $3,39,69$ \\
\hline
\end{tabular}

Notes. ${ }^{(\sharp)}$ PSRs B0105+68, B0643+80, B0656+14 were excluded from analysis because of RFI contamination.

References. [1] Bridle (1970); [2] Barr et al. (2013); [3] Bilous et al. (2016); [4] Boyles et al. (2013); [5] Bhat et al. (1999); [6] Bartel et al. (1978); [7] Champion et al. (2005); [8] Camilo \& Nice (1995); [9] Camilo et al. (1996); [10] Downs (1979); [11] Dembska et al. (2014); [12] Davies et al. (1977); [13] Deshpande \& Radhakrishnan (1992); [14] Downs et al. (1973); [15] Damashek et al. (1978); [16] Dewey et al. (1985); [17] Fomalont et al. (1992); [18] Gould (1994); [19] Han et al. (2009); [20] Hobbs et al. (2004); [21] Izvekova et al. (1979); [22] Izvekova et al. (1981); [23] Johnston et al. (2006); [24] Jankowski et al. (2018); [25] Kaplan et al. (1998); [26] Kijak et al. (2007); [27] Kramer et al. (1997); [28] Kijak et al. (1998); [29] Kuzmin et al. (1986); [30] Krzeszowski et al. (2014); [31] Kuz'min et al. (1978); [32] Karuppusamy et al. (2011); [33] Kramer et al. (1996); [34] Lane et al. (2014); [35] Löhmer et al. (2008); [36] Lewandowski et al. (2004); [37] Lorimer et al. (2005); [38] Lorimer et al. (1995); [39] Lommen et al. (2000); [40] Manchester (1971); [41] Malofeev (1993); [42] Malofeev (1999); [43] Morris et al. (1981); [44] Moffett \& Hankins (1999); [45] Murphy et al. (2017); [46] Maron et al. (2000); [47] Manchester et al. (1978); [48] Malofeev \& Malov (1980); [49] Malofeev et al. (2000); [50] Manchester \& Taylor (1981); [51] Navarro et al. (2003); [52] Rankin \& Benson (1981); [53] Rankin et al. (1970); [54] Ray et al. (1996); [55] Slee et al. (1986); [56] Stinebring \& Condon (1990); [57] Seiradakis et al. (1995); [58] Sayer et al. (1997); [59] Stovall et al. (2015); [60] Stokes et al. (1986); [61] Shrauner et al. (1998); [62] Sieber \& Wielebinski (1987); [63] Thorsett et al. (1993); [64] Taylor et al. (2000); [65] Vivekanand et al. (1983); [66] Wielebinski et al. (1993); [67] Wang et al. (2005); [68] Xue et al. (2017); [69] Zakharenko et al. (2013); [70] Zhao et al. (2017). 
Table B.1. continued.

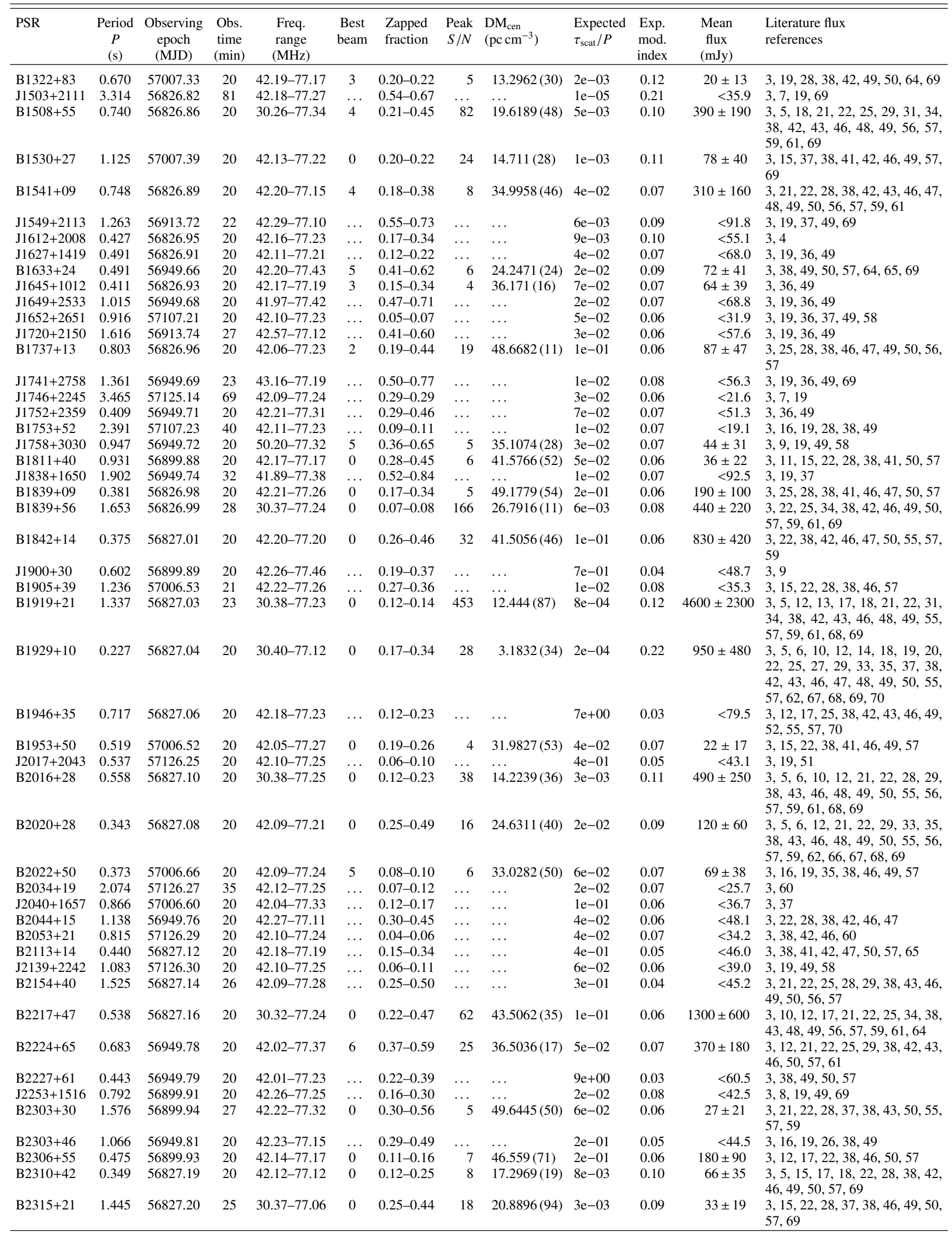


Table B.2. Fit results for pulsars with a single PL spectrum.

\begin{tabular}{|c|c|c|c|c|c|c|c|c|c|c|c|c|c|}
\hline PSR & $\begin{array}{c}\text { Frequency } \\
\text { span } \\
(\mathrm{MHz})\end{array}$ & $\begin{array}{l}\# \text { of } \\
\text { points, } \\
N_{p}\end{array}$ & $\begin{array}{r}\text { Ref. } \\
\text { freq., } \\
v_{0} \\
(\mathrm{MHz}) \\
\end{array}$ & $\begin{array}{r}\text { Ref. } \\
\text { flux, } \\
S_{0} \\
\text { (mJy) }\end{array}$ & $\begin{array}{c}\text { Spectral } \\
\text { index, } \\
\alpha\end{array}$ & $\begin{array}{c}\text { Fitted } \\
\text { flux } \\
\text { scatter, } \\
\sigma_{\lg S}^{\text {unkn }} \\
\end{array}$ & PSR & $\begin{array}{l}\text { Frequency } \\
\text { span } \\
(\mathrm{MHz})\end{array}$ & $\begin{array}{l}\# \text { of } \\
\text { points, } \\
N_{p}\end{array}$ & $\begin{array}{r}\text { Ref. } \\
\text { freq., } \\
v_{0} \\
(\mathrm{MHz}) \\
\end{array}$ & $\begin{array}{r}\text { Ref. } \\
\text { flux, } \\
S_{0} \\
(\mathrm{mJy})\end{array}$ & $\begin{array}{c}\text { Spectral } \\
\text { index, } \\
\alpha\end{array}$ & $\begin{array}{l}\text { Fitted } \\
\text { flux } \\
\text { scatter, } \\
\sigma_{\lg S}^{\text {unkn }} \\
\end{array}$ \\
\hline B $0011+47$ & $59-4850$ & 19 & 500 & 11.0 & $-0.9 \pm 0.1$ & 0.11 & $\mathrm{~J} 1238+21^{(\sharp)}$ & $25-430$ & 6 & 100 & 22.0 & $-0.8 \pm 0.3$ & . \\
\hline B $0053+47$ & $20-4850$ & 10 & 300 & 4.7 & $-1.3 \pm 0.2$ & 0.43 & $\mathrm{~J} 1313+0931_{(\sharp)}$ & $59-1400$ & 4 & 300 & 6.2 & $-2.3 \pm 0.3$ &. \\
\hline B $0226+70$ & $59-1420$ & 10 & 300 & 3.8 & $-1.6 \pm 0.2$ & 0.08 & $\mathrm{~J} 1645+1012^{(\sharp)}$ & $59-430$ & 4 & 200 & 14.0 & $-2.1 \pm 0.4$ & \\
\hline $\mathrm{J} 0611+30^{(\sharp)}$ & $45-430$ & 4 & 100 & 38.0 & $-1.9 \pm 0.4$ & $\ldots$ & $\mathrm{J} 1758+3030$ & $59-800$ & 9 & 200 & 20.0 & $-1.4 \pm 0.3$ & 0.11 \\
\hline B $0609+37$ & $59-4850$ & 13 & 500 & 5.2 & $-1.4 \pm 0.2$ & 0.30 & B $1842+14$ & $47-4850$ & 21 & 500 & 14.0 & $-1.95 \pm 0.09$ & 0.05 \\
\hline B $0655+64$ & $45-1408$ & 15 & 300 & 14.0 & $-2.0 \pm 0.2$ & 0.34 & $\mathrm{~B} 1953+50$ & $59-4850$ & 13 & 500 & 17.0 & $-1.2 \pm 0.1$ & 0.08 \\
\hline B $0751+32$ & $59-4850$ & 10 & 500 & 4.4 & $-1.4 \pm 0.2$ & 0.13 & $\mathrm{~B} 2022+50$ & $59-32000$ & 15 & 1400 & 1.9 & $-1.10 \pm 0.08$ & 0.07 \\
\hline B0917+63 & $45-1408$ & 10 & 300 & 6.3 & $-1.4 \pm 0.2$ & 0.07 & B $2224+65$ & $45-10700$ & 26 & 700 & 7.7 & $-1.62 \pm 0.07$ & 0.07 \\
\hline
\end{tabular}

Notes. ${ }^{(\sharp)}$ These pulsars have also broken PL fit, with break frequency fixed at the frequency of the largest measured flux density. See Table B.3 for the values of fitted parameters.

Table B.3. Fit results for pulsars where the spectrum was modelled with a broken PL.

\begin{tabular}{|c|c|c|c|c|c|c|c|c|c|}
\hline PSR & $\begin{array}{l}\text { Frequency } \\
\text { span } \\
(\mathrm{MHz})\end{array}$ & $\begin{array}{r}\# \text { of } \\
\text { points, } \\
N_{p}\end{array}$ & $\begin{array}{r}\text { Ref. } \\
\text { freq., } \\
v_{0} \\
(\mathrm{MHz}) \\
\end{array}$ & $\begin{array}{r}\text { Ref. } \\
\text { flux, } \\
S_{0} \\
(\mathrm{mJy})\end{array}$ & $\begin{array}{c}\text { Spectral } \\
\text { index, } \\
\alpha_{\text {lo }}\end{array}$ & $\begin{array}{r}\text { Break } \\
\text { freq., } \\
v_{\text {br }} \\
(\mathrm{MHz}) \\
\end{array}$ & $\begin{array}{c}\text { Uncertainty } \\
\text { range for } \\
v_{\mathrm{br}} \\
(\mathrm{MHz}) \\
\end{array}$ & $\begin{array}{c}\text { Spectral } \\
\text { index, } \\
\alpha_{\mathrm{hi}}\end{array}$ & $\begin{array}{l}\text { Fitted } \\
\text { flux } \\
\text { scatter, } \\
\sigma_{\lg S}^{\text {unkn }} \\
\end{array}$ \\
\hline B $0301+19$ & $59-4850$ & 35 & 500 & 24.0 & $-0.5 \pm 0.2$ & 500 & $354-761$ & $-1.9 \pm 0.3$ & 0.11 \\
\hline B $0320+39$ & $25-4850$ & 26 & 300 & 52.0 & $0.9 \pm 0.5$ & 157 & $133-195$ & $-2.4 \pm 0.2$ & 0.10 \\
\hline $\mathrm{J} 0611+30$ & $45-430$ & 4 & 100 & 70.0 & $1.4 \pm 1.5$ & 74 & & $-2.5 \pm 0.5$ & \\
\hline B $0809+74$ & $12-14800$ & 69 & 400 & 110.0 & $0.8 \pm 0.3$ & 66 & $58-73$ & $-1.66 \pm 0.06$ & 0.14 \\
\hline B $0943+10$ & $20-1400$ & 35 & 200 & 83.0 & $0.2 \pm 0.6$ & 114 & $87-167$ & $-2.8 \pm 0.7$ & 0.31 \\
\hline B $1112+50$ & $20-4900$ & 37 & 300 & 45.0 & $1.1 \pm 0.4$ & 148 & $133-172$ & $-2.3 \pm 0.2$ & 0.36 \\
\hline B $1133+16$ & $16-32000$ & 130 & 700 & 100.0 & $0.1 \pm 0.1$ & 232 & $212-257$ & $-1.93 \pm 0.06$ & 0.17 \\
\hline $\mathrm{J} 1238+21$ & $25-430$ & 6 & 100 & 64.0 & $0.8 \pm 0.5$ & 102 & $\ldots$ & $-2.4 \pm 0.4$ & \\
\hline $\mathrm{J} 1313+0931$ & $59-1400$ & 4 & 300 & 8.6 & $0.9 \pm 1.3$ & 149 & & $-2.6 \pm 0.3$ & \\
\hline B $1322+83$ & $25-1408$ & 12 & 200 & 59.0 & $0.8 \pm 0.6$ & 214 & $173-306$ & $-2.5 \pm 0.6$ & 0.18 \\
\hline B $1508+55$ & $20-10750$ & 59 & 500 & 52.0 & $2.4 \pm 0.4$ & 88 & $82-97$ & $-2.04 \pm 0.08$ & 0.14 \\
\hline B $1530+27$ & $25-4850$ & 22 & 300 & 17.0 & $1.3 \pm 0.5$ & 92 & $74-100$ & $-1.6 \pm 0.1$ & 0.05 \\
\hline B $1541+09$ & $39-10550$ & 49 & 600 & 29.0 & $0.7 \pm 0.4$ & 144 & $133-156$ & $-2.15 \pm 0.09$ & 0.09 \\
\hline B $1633+24$ & $25-1400$ & 12 & 200 & 44.0 & $0.6 \pm 0.5$ & 155 & $131-191$ & $-2.3 \pm 0.3$ & 0.06 \\
\hline $\mathrm{J} 1645+1012$ & $59-430$ & 4 & 200 & 21.0 & $1.5 \pm 1.7$ & 102 & & $-2.9 \pm 0.5$ & \\
\hline B $1737+13$ & $45-4850$ & 18 & 500 & 20.0 & $-0.6 \pm 0.5$ & 330 & $153-556$ & $-1.7 \pm 0.2$ & 0.04 \\
\hline B1839+09 & $59-4850$ & 13 & 500 & 14.0 & $-0.6 \pm 0.8$ & 229 & $135-294$ & $-1.9 \pm 0.2$ & 0.06 \\
\hline B $1839+56$ & $20-4850$ & 30 & 300 & 32.0 & $4.8 \pm 1.4$ & 39 & $35-41$ & $-1.6 \pm 0.1$ & 0.23 \\
\hline B $1919+21$ & $16-4850$ & 92 & 300 & 250.0 & $0.4 \pm 0.2$ & 135 & $120-149$ & $-2.7 \pm 0.1$ & 0.21 \\
\hline B $1929+10$ & $20-43000$ & 98 & 900 & 110.0 & $0.3 \pm 0.4$ & 342 & $240-511$ & $-1.74 \pm 0.09$ & 0.23 \\
\hline B2016+28 & $25-10700$ & 55 & 500 & 150.0 & $0.1 \pm 0.2$ & 331 & $293-379$ & $-2.27 \pm 0.09$ & 0.13 \\
\hline B $2020+28$ & $45-32000$ & 47 & 1200 & 24.0 & $0.6 \pm 0.6$ & 307 & $225-430$ & $-1.6 \pm 0.1$ & 0.21 \\
\hline B2306+55 & $59-4850$ & 13 & 500 & 15.0 & $-0.7 \pm 0.5$ & 357 & $178-532$ & $-2.0 \pm 0.2$ & 0.07 \\
\hline B $2310+42$ & $25-10700$ & 34 & 500 & 130.0 & $0.1 \pm 0.3$ & 645 & 498-909 & $-2.1 \pm 0.3$ & 0.18 \\
\hline B $2315+21$ & $25-4850$ & 16 & 400 & 17.0 & $0.6 \pm 0.5$ & 186 & $167-231$ & $-2.1 \pm 0.2$ & 0.06 \\
\hline
\end{tabular}

Table B.4. Fit results for pulsars where the spectrum was modelled by a PL with two breaks.

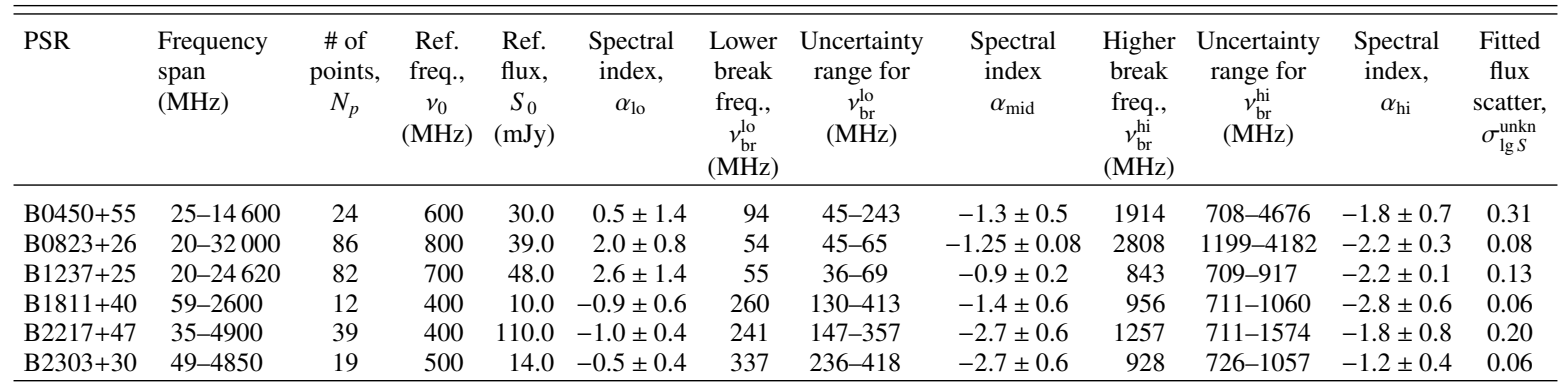




\section{Appendix C: Profiles and spectra}
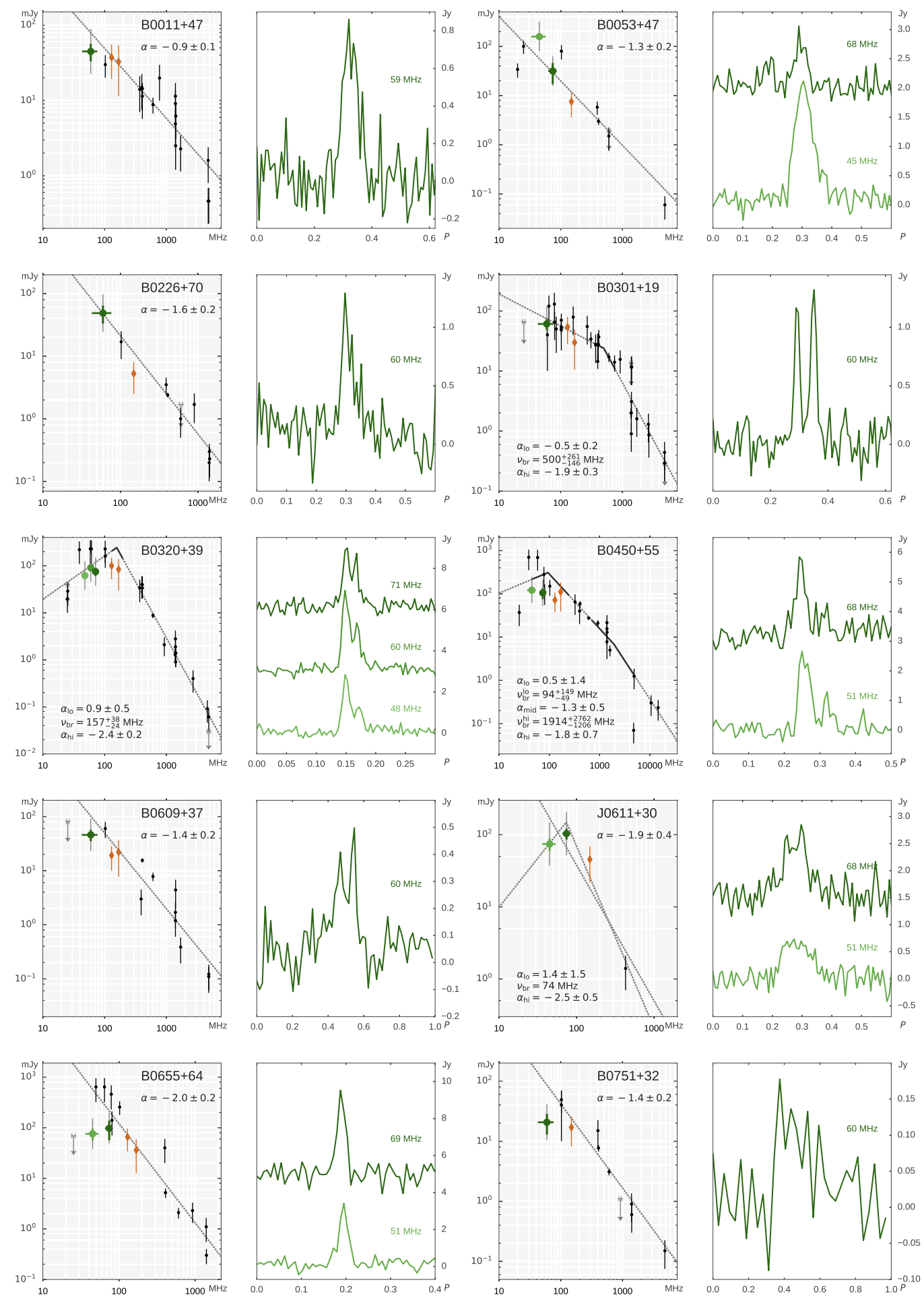

Fig. C.1. For each pair of plots, left: spectra of radio emission for pulsars detected in the census. Smaller black points with error bars mark literature flux densities, larger green dots show LOFAR LBA census measurements at various frequencies (with horizontal errorbars indicating the frequency span of a given census measurement), brown diamonds show flux densities from HBA census (B16), and arrows show upper limits. For LBA census measurements, thin grey errorbars show $\pm 50 \%$ flux uncertainty and thicker green ones show uncertainty due to limited S/N. See text for both census and literature flux density errors and upper limit discussion. In the case of a multiple-PL fit, the uncertainty on the break frequency is marked with a broken black line. Right: flux-calibrated average profiles for LOFAR LBA census observations. Multiple profiles per band are shown with a constant flux offset between separate subbands. The choice of the number of subbands was defined by the peak $\mathrm{S} / \mathrm{N}$ of the average profile, the presence of profile evolution within the observing band and the number of previously published flux density values. 
A. V. Bilous et al.: A LOFAR census of non-recycled pulsars, LBA
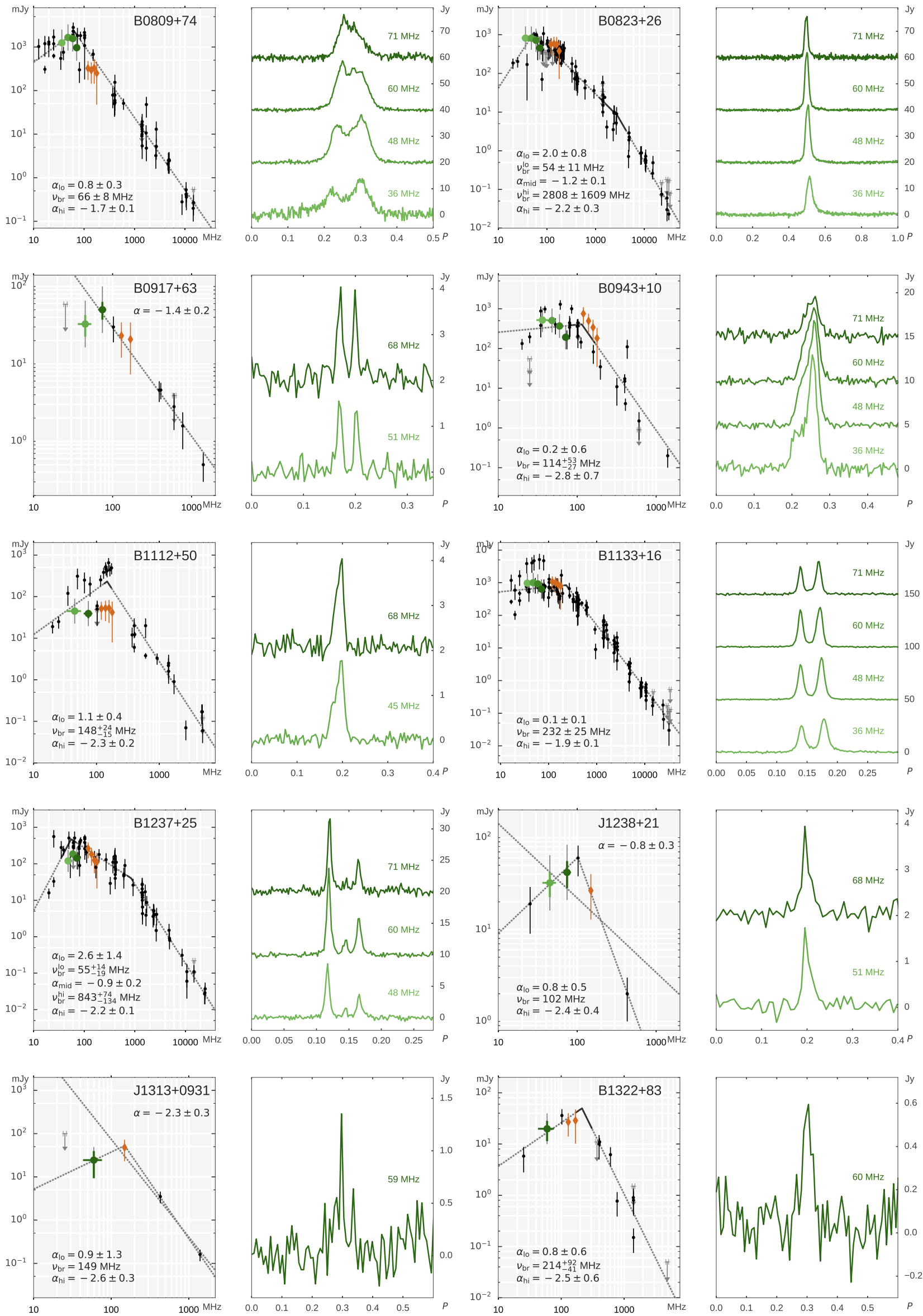

Fig. C.1. continued. 

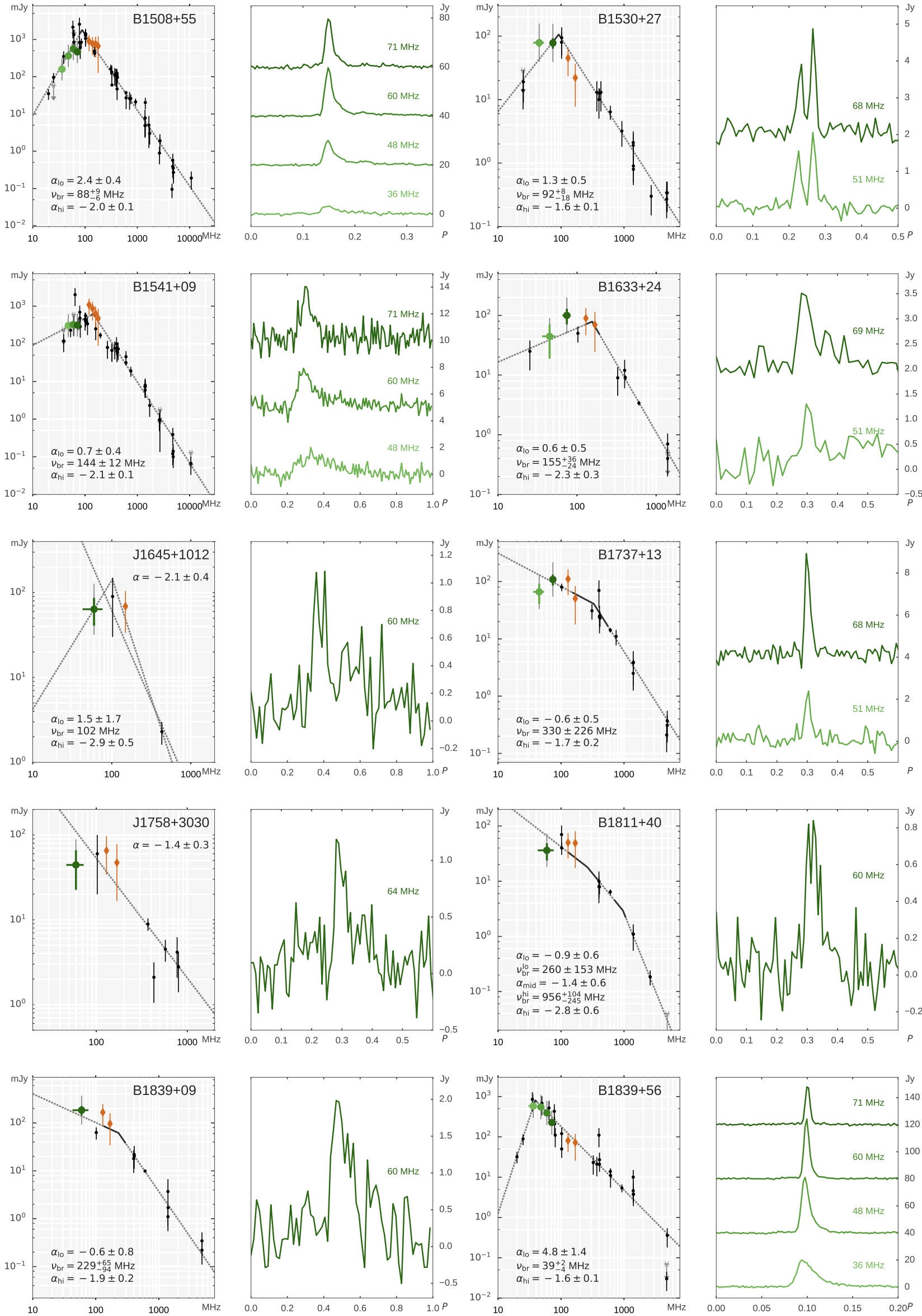

Fig. C.1. continued. 
A. V. Bilous et al.: A LOFAR census of non-recycled pulsars, LBA
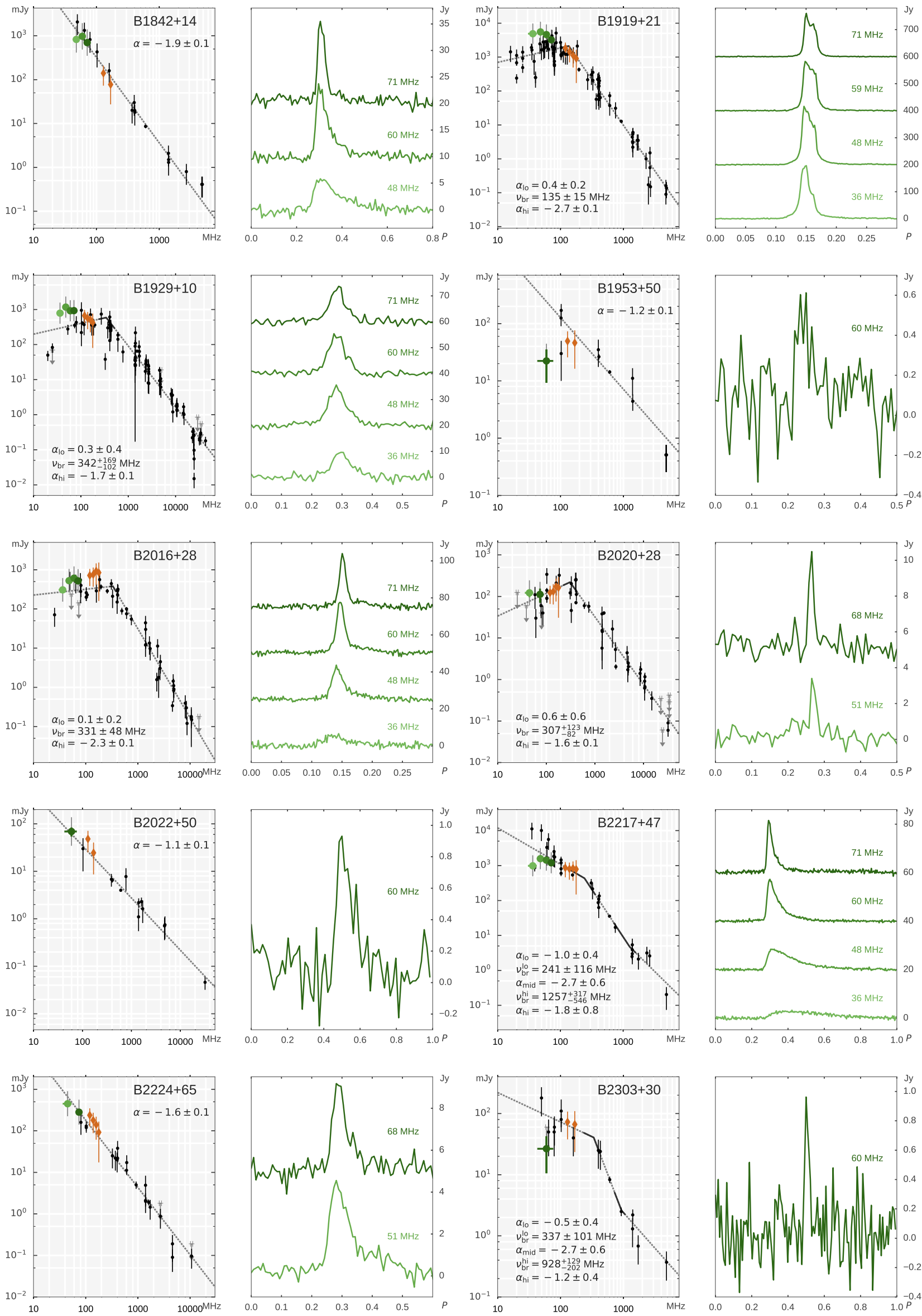

Fig. C.1. continued. 
A\&A 635, A75 (2020)
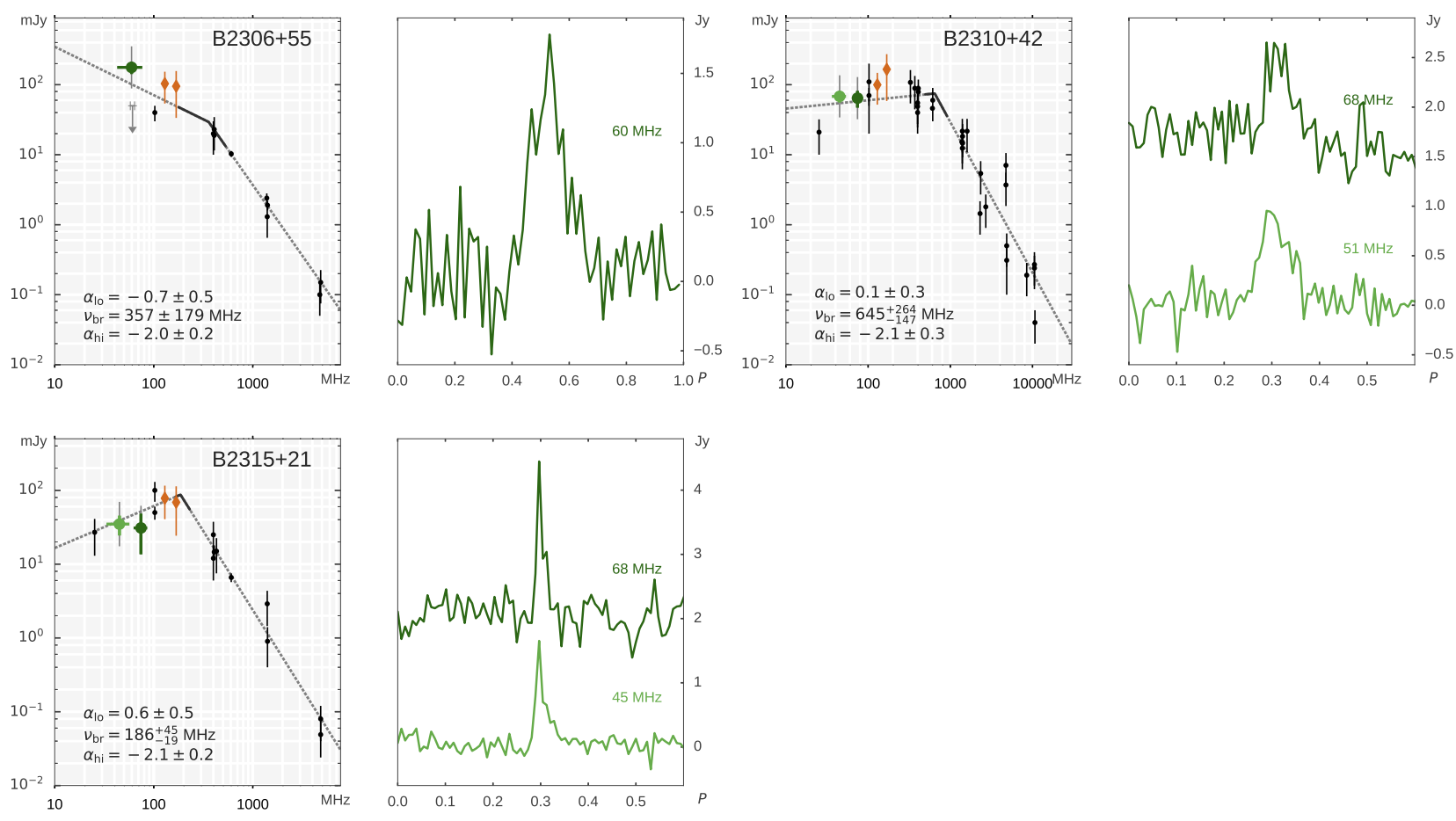

Fig. C.1. continued. 